\title{
Comparing The Efficacy of Anti-Infectious Drugs For The Treatment of Mild To Severe COVID-19 Patients: A Protocol For A Systematic Review And Network Meta-Analysis of Randomized Clinical Trials.
}

Dejene Tolossa Debela ( $\square$ dejene.tolossa@aau.edu.et )

Addis Ababa University College of Health Sciences https://orcid.org/0000-0002-4615-5839

Kidist Digamo Heraro

Addis Ababa University College of Health Sciences

Abebaw Fekadu

Addis Ababa University College of Health Sciences

Merga Belina

Addis Ababa University College of Health Sciences

Tsegahun Manyazewal

Addis Ababa University College of Health Sciences

\section{Protocol}

Keywords: network meta-analysis, systematic review, covid-19, anti-viral agents

Posted Date: June 15th, 2021

DOI: https://doi.org/10.21203/rs.3.rs-585756/v1

License: (c) (1) This work is licensed under a Creative Commons Attribution 4.0 International License.

Read Full License 


\section{Abstract}

Background: COVID-19 is a viral infection spreading at a great speed and has quickly caused an extensive burden to individuals, families, countries, and the world. No intervention has yet been proven highly effective for the treatment of COVID-19. Different drugs were being evaluated and reported through randomized clinical trials, and more are currently under trial. This review aimed to compare the efficacy of anti-infectious drugs with a comparator of the standard of care or placebo in patients with COVID-19.

Methods: Two independent review authors will extract data and assess a risk of bias using RoB2. Randomized controlled trials (RCT) that evaluate single and/or combined antiviral drugs recommended by WHO latest guideline for the treatment of COVID-19 will be included. We will search for Pub Med, the Cochrane Center for Clinical Trial database (CENTRAL), clinicaltrials.gov, etc. databases for articles published in the English language between December 2019 to April 2021. We will follow the Preferred Reporting Items for Systematic Review and Meta-Analysis Protocols (PRISMA-P) involving Network Metaanalysis guidelines to design and report of the results. The primary endpoints will be time to clinical recovery and time to RNA negativity. The certainty of evidence will be evaluated using the GRADE extension of NMA. Data analysis will be performed using the frequentist NMA approach with the netmeta package implemented in $\mathrm{R}$.

Discussion: This review will reveal the best antiviral drug treatment for covid-19 and show the hierarchy of those drugs.

Systematic review registration: The protocol was registered on PROSPERO with ID number CRD42021230919

\section{Background}

In China, in December 2019, a novel coronavirus named severe acute respiratory syndrome coronavirus 2 (SARS CoV-2) caused an international outbreak of a respiratory illness called coronavirus disease 2019 [COVID-19](1). Since then, SARS-CoV-2 has spread globally, and COVID-19 has now been labeled a pandemic of international concern by the World Health Organization(2).

Coronaviruses are enveloped, positive-sense, single-stranded RNA virus genomes. The coronavirus encodes a nonstructural replicase polyprotein and structural proteins, including spike (S), envelope $(E)$, membrane (M), and nucleocapsid $(\mathrm{N})(3-5)$. The S protein on the surface of SARS-CoV is the most common target for the development of vaccines and therapeutics(6).

There are about thirty types of coronaviruses infecting mammals, birds, and other animals. Only seven of them infect humans $(4,7)$. Four of them usually cause mild diseases such as the common cold (HKU1; OC43; 229E; and NL63), whereas MERS-CoV, SARS-CoV, and now SARS-CoV-2 are likely to cause more serious diseases $(5,7)$. The main transmission way of SARS-CoV is from human to human by respiratory droplets(8-10). 
COVID-19 disease clinical presentation can be from subclinical infection with mild (self-limiting respiratory tract illness) to severe (progressive pneumonia, multiorgan failure, and death) (11-14). Massive alveolar damage and progressive respiratory failure are the cause of death in severe covid-19 disease (12). Patients having comorbidities like people with chronic lung disease, serious heart disease, chronic kidney disease, elderly (above 65 years), and immunocompromised people are suspected to have the severe disease(15).

As of November 04,2020 , there were $47,362,304$ confirmed patients, $1,211,986$ confirmed deaths, and 219 countries, areas, or territories with COVID-19 according to the World Health Organization(16).

The only anti-viral drug FDA approved is Remdesivir yet for the treatment of COVID-19 in hospitalized patients (aged $\geq 12$ years and weighing $\geq 40 \mathrm{~kg}$ )(17). To control the growing COVID-19 pandemic, we rely on quarantine, isolation, and infection-control measures preventing the spread of disease as well as oxygen and mechanical ventilation as supportive care for infected patients(18). Currently, there are many drugs exist that are being under assessment for patients with COVID-19: example, Remdesivir (used to treat Ebola virus disease and Marburg virus infections), lopinavir and ritonavir (used to treat HIV/AIDS), chloroquine phosphate or hydroxychloroquine (used to treat malaria), tocilizumab (used to treat rheumatoid arthritis), corticosteroids, stem cells, and other types of interventions(19).

Several randomized clinical trials are underway. According to an online global COVID-19 clinical trial tracker available at www.covid19-trials.org, there are currently 2462 trials registered worldwide as about $20 \%$ of them are in the US.

Although the mortality rate is concerning, the high transmissibility of the disease is much more alarming. Even if a low percentage of patients need hospitalization, the rapid spread of the disease and a large number of people infected has overwhelmed the healthcare systems worldwide. To decrease the spread, severe social distancing measures, travel restrictions, closures of schools, and many businesses are taking an unprecedented socioeconomic and psychological toll. Therefore, COVID-19 has caused an enormous impact on people's quality of life and posed far-reaching threats, especially to the economy, health, and the sustainability of healthcare systems(18).

There have been many efforts done to identify effective drug treatment for covid-19 but, evidence for effective treatment remains limited. It is, therefore, an urgent need of investigating the most effective drugs to slow the progression of the disease and unburden the health care systems. Although extraordinary efforts have been made on research regarding pharmacological interventions, none have proven most effective. Therefore, this systematic review and network meta-analysis aim to synthesize existing evidence to compare the efficacy and safety as well as identify the best drug among different anti-infectious drug categories for the treatment of mild to severe patients with COVID-19.

\section{Methods}


This research is designed and will be reported by a systematic review involving a network meta-analysis that will comply with the Preferred Reporting Items for Systematic Review and Meta-Analysis Protocols (PRISMA-P) 2015 guidelines(20) (see Additional file 1). This protocol has been registered at the PROSPERO 2021 database, ID = CRD42021230919.

\section{Data sources and searches}

We will search databases from December 2019 to April 2021. We will search PubMed, the Cochrane Center for Clinical Trial database (CENTRAL), clinicaltrials.gov, clinical trials register.EU, chictr.org.cn, covid-19.cochrane.org, and covid-evidence.org. databases for articles published worldwide in the English language. The search will be done according to guidance provided in the Cochrane Handbook for Systematic Reviews of Interventions. The search will be limited to human studies, published in English languages until April 2021. We will check for any unidentified randomized clinical trials from reference lists of relevant trial publications. The authors of the included trials will be contacted by email asking for unpublished randomized clinical trials. We will do a thorough search strategy using the Mesh terms and keywords including SARS-CoV-2, COVID-19, COVID-19 serotherapy, SARS-CoV-2 variants, Antiviral Agents, Hydroxychloroquine, Remdesivir, GS-441524, Lopinavir, Lopinavir-ritonavir drug combination, favipiravir, Ivermectin, chloroquine, azithromycin, Randomized Controlled Trial and RCT (Table 1).

Table 1

search string for PubMed database

$\begin{array}{ll}\text { SN } & \text { Search terms } \\ \text { \#1 } & \text { "Antiviral Agents"[Mesh] OR "Antiviral Agents" [Pharmacological Action] OR } \\ & \text { "Hydroxychloroquine"[Mesh] OR "remdesivir" [Supplementary Concept] OR "GS- } \\ & 441524 \text { " [Supplementary Concept] OR "Lopinavir"[Mesh] OR "lopinavir-ritonavir drug } \\ & \text { combination" [Supplementary Concept] OR "favipiravir" [Supplementary Concept] OR } \\ & \text { "Ivermectin"[Mesh] }\end{array}$

\#2 $\quad$ "SARS-CoV-2"[Mesh] OR "COVID-19"[Mesh] OR "COVID-19 serotherapy" [Supplementary Concept] OR "SARS-CoV-2 variants" [Supplementary Concept]

\#3 $\quad$ "Randomized Controlled Trial" [Publication Type] OR "Randomized Controlled Trials as Topic"[Mesh]

\#4 \#1 AND \#2 AND \#3

Summarizes the search strategy that we will use in the PubMed database.

Eligibility criteria

We will identify eligible studies through the PICOS (participants, interventions, comparison, outcomes, and study designs) format(21)

\section{Types of participants}


We will include all patients diagnosed with laboratory-confirmed, mild to severe COVID 19 patients of both sexes and all ages with any comorbidities.

\section{Types of interventions}

We will include any anti-infectious drugs used to treat COVID-19 recommended by WHO's latest guideline including Hydroxychloroquine, Remdesivir, Lopinavir, Lopinavir-ritonavir, favipiravir, Ivermectin, chloroquine, azithromycin, etc.

\section{Types of comparators}

We will include the standard of care, placebo, or another drug group.

\section{Types of outcome measures}

\section{Primary outcome:}

- Time to clinical improvement and time to viral clearance (RNA negativity)

\section{Secondary outcomes}

- Mortality rate, length of hospital stays, rate of patients' needs for oxygen therapy, and adverse events $(\mathrm{AE})$.

\section{Types of studies}

We will include only randomized clinical trials (RCT) that compared the efficacy of anti-infectious drugs for the treatment of COVID 19 against standard care or placebo or other medication.

\section{Selection of studies}

Endnote software version X7 will be used to import the research articles from the electronic databases and duplicates will be removed. Two review authors will independently screen titles and abstracts based on pre-specified eligibility criteria and retrieve all relevant full-text study reports. Any disagreements between two review authors will be resolved through discussion, or if required, they will consult a third person. The screening and selection process will be reported in a PRISMA flow chart as summarized by Fig. 1.

\section{Data extraction}

The data will be extracted independently by 2 reviewers using a predefined format. Disagreements will be resolved by discussion. The two review authors will evaluate all available data simultaneously to maximize data extraction by assessing duplicated publications and companion papers of a trial together. We will name each trial after the first author and year of the publication. The information will be collected include participants (demographic and clinical characteristics), the pharmacological treatment (name of 
the drug, treatment duration, dose), time points used for the assessments, number of patients lost (in each group), reasons for loss to follow-up, missing data (intention-to-treat or per protocol), sources of funding, possibility of a conflict of interests, adverse events, outcome measures (primary and secondary outcomes), protocol deviations. Also, Relevant information such as title, author name, year of publication, publication status, study design, study setting, follow-up period, sample size, funding of the trial or sources of support, baseline characteristics of study subjects, will be extracted. The trial authors will be contacted by email to specify any missing data, which may not be reported sufficiently or not at all in the publication.

\section{Assessment of risk of bias}

The risk of bias assessment will be based on the Cochrane Risk of Bias tool version 2 (Rob 2) as recommended in The Cochrane Handbook of Systematic Reviews of Interventions(22). We will evaluate the methodology to reduce the risk of bias across the following five domains (Bias from the randomization process, deviation from intended interventions, missing outcome data, measurement of outcomes, and selective reporting of results). The risk of bias of each trial will be judged by two independent authors as low risk, some concerns, and a high risk of bias. The disagreements will be resolved by discussion between the two authors.

\section{Statistical analysis}

We planned to do all statistical analyses using $\mathrm{R}$ version 4.0.3 software for Window 10. Odds ratios (OR) with a $95 \%$ confidence interval $(\mathrm{Cl})$ for dichotomous outcomes and mean differences (MDs) with $95 \% \mathrm{Cl}$ for continuous outcomes data will be used to estimate the relative treatment effects of the competing interventions. We will convert other forms of data into MDs using the standard conversion formula. For outcome variables reported in different scales, we will use standard mean differences with $95 \% \mathrm{Cls}$. Other binary outcome data will be converted into OR. Estimation of the ranking probabilities will be done for all included anti-infectious drugs being at each possible rank for each intervention. The surface under the cumulative ranking curve (SUCRA) and mean ranks will be used to obtain a treatment hierarchy.

\section{Meta-analysis and network meta-analysis}

We will undertake the meta-analyses according to the Cochrane Handbook of Systematic Reviews of Interventions(23) using R version 4.0.3 software for every treatment comparison with at least two studies. Effect sizes of individual studies and any pooled estimates of effect will be presented in tables and graphically as forest plots. We will use forest plots to visually evaluate any sign of heterogeneity. Then we assess the presence of statistical heterogeneity using the 12 statistic. Substantial heterogeneity $\left(I^{2}>\right.$ $50 \%)(24-26)$. We planned to perform network meta-analysis using a frequentist NMA approach and a random-effects model for each treatment comparison, using the netmeta package version 1.2-1 implemented in R version 4.0.3 software for Window 10, if the assumption of transitivity is fulfilled. We will categorize the network nodes as follows: 1. Chloroquine, 2. Hydroxychloroquine 3.

Lopinavir/Ritonavir, 4. Remdesivir, 5. Ivermectin 6. Favipiravir, 7. The combinations of those drugs. We 
will assess inconsistency globally across the whole network. If evidence of inconsistency is found, we will assess locally (a node splitting approach) to identify possible areas of local inconsistency and, if sufficient data exist, run network meta-regression. We will extend the analysis to all closed loops assuming a loop-specific heterogeneity and examine the estimates of inconsistency together with $95 \%$ confidence intervals for each loop using a graphical representation. Any orderings of treatment hierarchy will be estimated in primary outcomes and present treatment rankings with P-score using the Net rank of the R package netmeta.

We will conduct a sensitivity analysis for all studies assessed as being low risk of bias and high risk of bias to test the robustness of our data. We will do subgroup analysis using network meta-regression in the primary outcomes influenced by available variables: age, sex, comorbidities, and disease severity (mild, moderate, and severe).

Confidence in the evidence will be assessed using the GRADE working group recommendations and the CINeMA software and classify evidence as high, moderate, low, or very low certainty $(27,28)$. This rating method follows steps required are from 1) direct, 2) indirect, 3) NMA evidence, and 4) direct and indirect comparisons. Assessments of the evidence will be presented using the six domains: (study limitations, indirectness, inconsistency, imprecision, publication bias, incoherence)(28). The results will be presented in an 'NMA SoF table.

\section{Dealing with missing data}

We will contact all trial authors to obtain clarification for any relevant missing data. If the authors will not respond imputation method will be used.

\section{Reporting bias}

We will use the comparison-adjusted and contour-enhanced funnel plot and Egger's test to visually assess publication bias if ten or more trials will be included(24). We also use the adjusted rank correlation $(29,30)$.

\section{Abbreviations}

ACE2-_____-_-_ngiotensin-Converting Enzyme 2

AE-_______-_Adverse Event

COVID-19-____-_Corona Virus Disease 2019

FDA-_-_-_-_ood and Drug Agent

GRADE-_-_-_-Grading of Recommendations Assessment, Development, and Evaluation

HIV/AIDS-_____Human Immune Virus Acquired IIIness Disease Syndrome 
MeSH-___-_-_Medical Subject Heading

MD_-_-_Mean Difference

MERS-CoV_____-Middle East Respiratory Syndrome Corona Virus

PRISMA_______Preferred Reporting Items for Systematic Review and Meta-Analysis

RCT-________nandomized Clinical Trial

RNA_-_-_Ribosomal Nucleic Acid

RoB2-______ Risk of Bias tool version 2

SARS-CoV-2-_-_-_Severe Acute Respiratory Syndrome Corona Virus 2

SLE-_-_Systemic Lupus Erythematosus

TMPRSS2-_____-_Transmembrane Serine Protease 2

WHO_-_World Health Organization

3CLpro-_-_-_-_Chymotrypsin-Like Protease

\section{Declarations}

Ethics approval and consent to participate: There are no ethical considerations associated with this study as we will use publicly available data from previously published studies.

Consent for publication: We planned to publish results in open access peer reviewed journals.

Availability of data and materials: Not applicable.

Competing interests: The authors declare that they have no competing interests

Funding: None

Authors' contributions: DTD conceived the content, wrote the paper, and approved the final version. All other authors conducted the preliminary search, copyedited and revised the manuscript. All authors have read and approved the manuscript.

Acknowledgements: Not applicable

\section{References}


1. Guan W-j, Ni Z-y, Hu Y, Liang W-h, Ou C-q, He J-x, et al. Clinical Characteristics of Coronavirus Disease 2019 in China. N Engl J Med. 2020;382(18):1708-20.

2. Organization WH. Novel Coronavirus ( 2019-nCoV): situation report, 3. 2020.

3. Du L, He Y, Zhou Y, Liu S, Zheng B-J, Jiang S. The spike protein of SARS-CoV-a target for vaccine and therapeutic development. Nat Rev Microbiol. 2009;7(3):226-36.

4. Li G, Fan Y, Lai Y, Han T, Li Z, Zhou P, et al. Coronavirus infections and immune responses. Journal of medical virology. 2020;92(4):424-32.

5. Masters PS. The molecular biology of coronaviruses. Adv Virus Res. 2006;66:193-292.

6. Bisht H, Roberts A, Vogel L, Bukreyev A, Collins PL, Murphy BR, et al. Severe acute respiratory syndrome coronavirus spike protein expressed by attenuated vaccinia virus protectively immunizes mice. Proceedings of the National Academy of Sciences. 2004;101(17):6641-6.

7. Zhu N, Zhang D, Wang W, Li X, Yang B, Song J, et al. A novel coronavirus from patients with pneumonia in China, 2019. New England Journal of Medicine; 2020.

8. Covid C, COVID C, COVID C, Bialek S, Gierke R, Hughes M, et al. Coronavirus Disease 2019 in Children -United States, February 12-April 2, 2020. Morb Mortal Wkly Rep. 2020;69(14):422.

9. Ali M, Shah STH, Imran M, Khan A. The role of asymptomatic class, quarantine and isolation in the transmission of COVID-19. J Biol Dyn. 2020;14(1):389-408.

10. Organization WH. Report of the WHO-China Joint Mission on coronavirus disease 2019 (COVID-19). 2020. Significant account of fatality rates and comorbidities in reports from China related to COVID19 infection. 2020.

11. Cao B, Wang Y, Wen D, Liu W, Wang J, Fan G, et al. A trial of lopinavir-ritonavir in adults hospitalized with severe Covid-19. New England Journal of Medicine; 2020.

12. Huang C, Wang Y, Li X, Ren L, Zhao J, Hu Y, et al. Clinical features of patients infected with 2019 novel coronavirus in Wuhan, China. The lancet. 2020;395(10223):497-506.

13. Chen N, Zhou M, Dong X, Qu J, Gong F, Han Y, et al. Epidemiological and clinical characteristics of 99 cases of 2019 novel coronavirus pneumonia in Wuhan, China: a descriptive study. The Lancet. 2020;395(10223):507-13.

14. Wang D, Hu B, Hu C, Zhu F, Liu X, Zhang J, et al. Clinical characteristics of 138 hospitalized patients with 2019 novel coronavirus-infected pneumonia in Wuhan, China. Jama. 2020;323(11):1061-9.

15. Control CfD. Prevention. Groups at Higher Risk for Severe Illness. 2020. 2020.

16. Organization WH. Coronavirus disease (COVID-19) outbreak. 2020. URL https://www who int/emergencies/diseases/novel-coronavirus-2019. 2020.

17. Food U, Administration D. FDA Approves First Treatment for COVID-19. 2020.

18. Sohrabi C, Alsafi Z, O'Neill N, Khan M, Kerwan A, Al-Jabir A, et al. World Health Organization declares global emergency: A review of the 2019 novel coronavirus (COVID-19). International Journal of Surgery. 2020. 
19. Organization WH. WHO R\&D Blueprint: informal consultation on prioritization of candidate therapeutic agents for use in novel coronavirus 2019 infection, Geneva, Switzerland, 24 January 2020. World Health Organization; 2020.

20. Hutton B, Catala-Lopez F, Moher D. The PRISMA statement extension for systematic reviews incorporating network meta-analysis: PRISMA-NMA. Med Clin (Barc). 2016;147(6):262-6.

21. McKenzie JEBS, Ryan RE, Thomson HJ, Johnston RV, Thomas J. Defining the criteria for including studies and how they will be grouped for the synthesis In: Higgins JPT TJ, Chandler J, Cumpston M, Li T, Page MJ, Welch VA editor. Cochrane Handbook for Systematic Reviews of Interventions version 60 (updated. July. 2019)2019.

22. Sterne JAC, Savović J, Page MJ, Elbers RG, Blencowe NS, Boutron I, et al. RoB 2: a revised tool for assessing risk of bias in randomised trials. BMJ. 2019;366:14898.

23. Higgins JPTJ, Chandler J, Cumpston M, Li T, Page MJ, Welch VA, editors. Cochrane handbook for systematic reviews of interventions. John Wiley \& Sons; 2019.

24. Higgins JP, Thomas J, Chandler J, Cumpston M, Li T, Page MJ, et al. Cochrane handbook for systematic reviews of interventions. John Wiley \& Sons; 2019.

25. Higgins JP, Thompson SG. Quantifying heterogeneity in a meta-analysis. Statistics in medicine. 2002;21(11):1539-58.

26. Higgins JP, Thompson SG, Deeks JJ, Altman DG. Measuring inconsistency in meta-analyses. BMJ. 2003;327(7414):557-60.

27. Salanti G, Del Giovane C, Chaimani A, Caldwell DM, Higgins JP. Evaluating the quality of evidence from a network meta-analysis. PLoS One. 2014;9(7):e99682.

28. Nikolakopoulou A, Higgins JP, Papakonstantinou T, Chaimani A, Del Giovane C, Egger M, et al. Assessing Confidence in the Results of Network Meta-Analysis (Cinema). bioRxiv. 2019:597047.

29. Egger M, Smith GD, Schneider M, Minder C. Bias in meta-analysis detected by a simple, graphical test. BMJ. 1997;315(7109):629-34.

30. Begg CB, Mazumdar M. Operating characteristics of a rank correlation test for publication bias. Biometrics. 1994:1088 - 101.

\section{Figures}




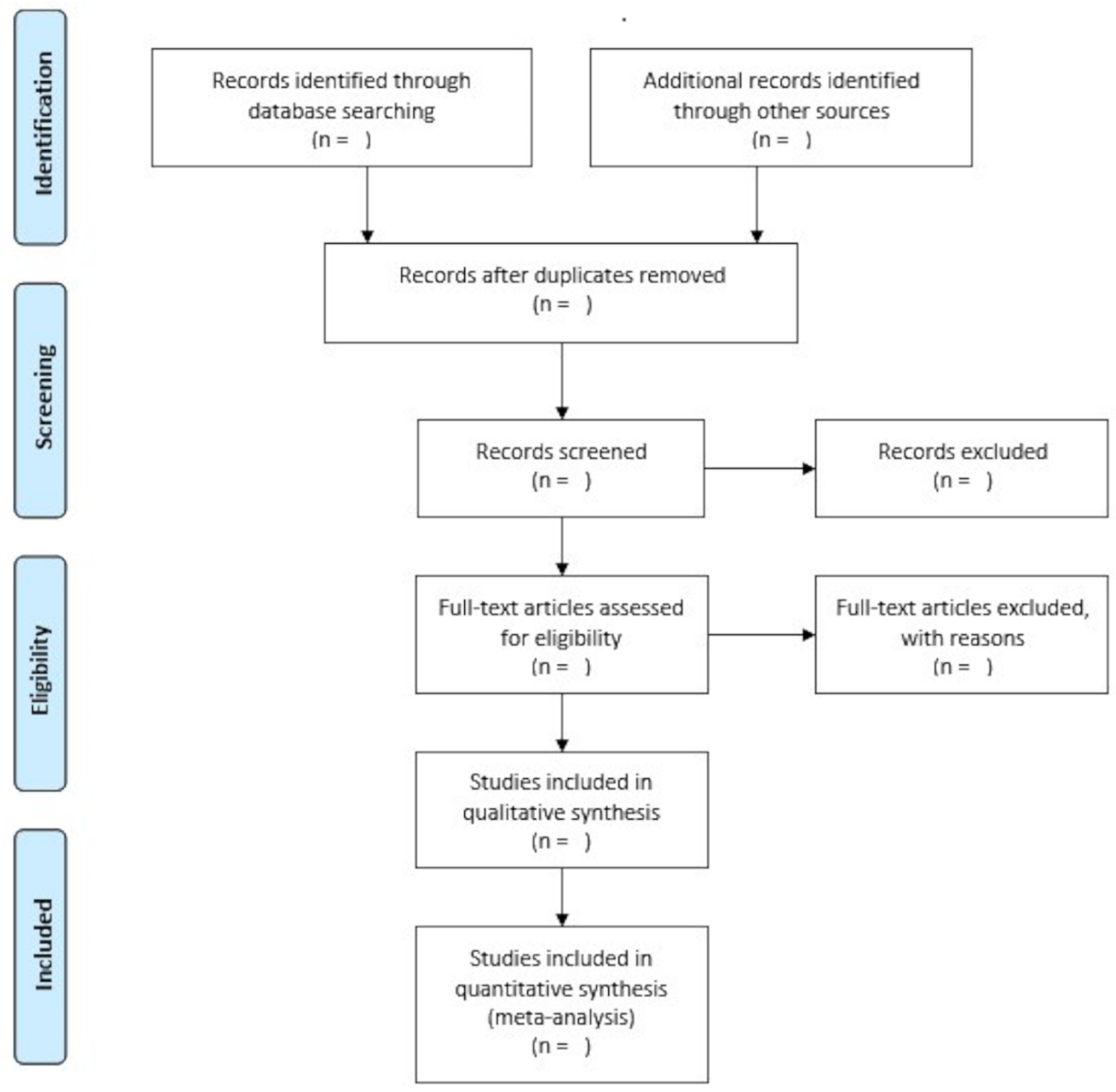

Figure 1

PRISMA flow diagram chart showing the process of study selection. Modified from Cochrane.

\section{Supplementary Files}

This is a list of supplementary files associated with this preprint. Click to download.

- PRISMAPchecklist.docx 